\title{
Preparation of N-substituted sulfoximines by benzotriazole methodology
}

\author{
Alan R. Katritzky,* Yuming Zhang, Sandeep K. Singh, and Yves P. Le Gall \\ Center for Heterocyclic Compounds, Department of Chemistry, \\ University of Florida, Gainesville, FL 32611-7200 \\ E-mail: Katritzky@chem.ufl.edu \\ (received 13 Nov 03; accepted 09 Jan 04; published on the web 15 Jan 04)
}

\begin{abstract}
Diverse $N$-substituted sulfoximines $\mathbf{5 a - n}$ were prepared by nucleophilic replacement of the benzotriazole moiety in $N$-(benzotriazol-1-ylalkyl)sulfoximines 3a-e using organozinc reagents or allylsilanes. $\mathrm{N}$-(Benzotriazol-1-ylalkyl)sulfoximines 3 , in turn, were obtained by condensation of sulfoximines 1 with aldehydes 2 and benzotriazole.
\end{abstract}

Keywords: N-Substituted sulfoximines, condensation, nucleophilic substitution, organozinc reagents, allylsilanes

\section{Introduction}

$N$-Functionalized sulfoximine derivatives are antimuscarinic, spasmolytic, ${ }^{1}$ antiarrhythmic, ${ }^{2}$ $\gamma$-glutamylcysteine synthetase inhibitors, ${ }^{3}$ possess antitumor activity, ${ }^{4}$ and are important synthetic intermediates. ${ }^{5}$ Several methods have been developed for the preparation of $N$-substituted sulfoximines from $\mathrm{NH}$-sulfoximines: (i) Eschweiler-Clark conditions for $\mathrm{N}$-methylated sulfoximines; ${ }^{6}$ (ii) palladium-catalyzed reactions for $N$-arylated sulfoximines; ${ }^{7}$ and (iii) base-catalyzed Michael-type additions ${ }^{8}$ or base-promoted alkylations ${ }^{9 \mathrm{a}}$ or acylations ${ }^{9 \mathrm{~b}}$ (Scheme 1).

Nucleophilic substitution of the benzotriazole moiety in benzotriazolylmethyl amines is an efficient method to prepare $N$-alkylated amines, ${ }^{10}$ amides, ${ }^{11}$ thioamides, ${ }^{12}$ or sulfonamides. ${ }^{13}$ Herein, we report the preparation of $N$-(benzotriazol-1-ylalkyl)sulfoximines 3 as intermediates and subsequent nucleophilic replacement of the benzotriazolyl anion to introduce a simple route to $N$-substituted sulfoximines 5 .

\section{Results and Discussion}

\section{Preparation of $\boldsymbol{N}$-(benzotriazol-1-ylmethyl)sulfoximines 3a-e}

A variety of benzotriazolyl intermediates, which provide convenient routes to diverse heterocycles, ${ }^{14 a}$ are readily available by condensations of benzotriazole and aldehydes with amides, thio 


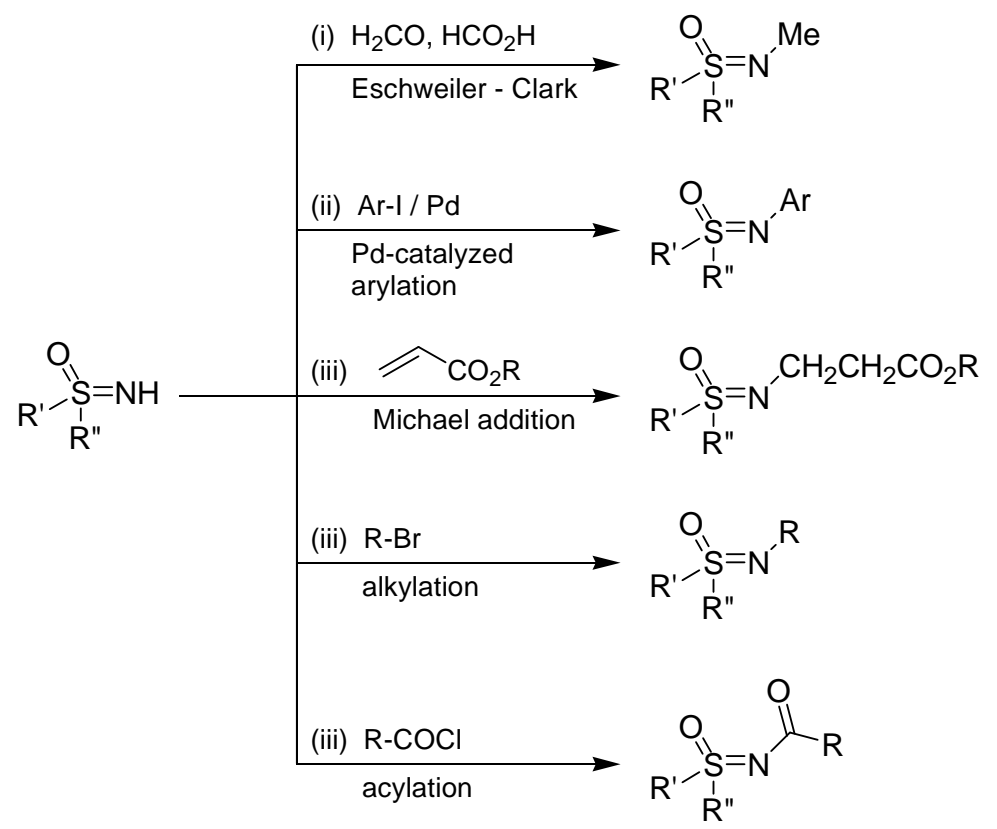

\section{Scheme 1}

amides, sulfonamides or acylhydrazines. ${ }^{14 \mathrm{~b}}$ We have now similarly prepared $N$-functionalized sulfoximines via $N$-(benzotriazol-1-ylmethyl)sulfoximines 3a-e (Scheme 2, Table 1). Thus, condensation of $( \pm)-S$-methyl-S-phenylsulfoximine (1a) with formaldehyde and benzotriazole in the presence of catalytic amounts of $p$-toluenesulfonic acid in refluxing toluene gave $N$-(benzotriazol-1-ylmethyl)-S-methyl-S-phenylsulfoximine (3a) in $73 \%$ yield. Similarly, reaction of 1a with benzotriazole and ethyl glyoxylate gave the desired ethyl 2-(1H-1,2,3benzotriazol-1-yl)-2-[[methyl(oxo)phenyl- $\lambda^{6}$-sulfanylidene]amino]acetate (3b) in $82 \%$ yield. Condensation of diphenyl sulfoximine with formaldehyde or ethyl glyoxylate and benzotriazole gave the corresponding benzotriazole adducts $3 \mathbf{c}$ and $\mathbf{3 d}$ in $78 \%$ and $71 \%$ yield, respectively. Use of $(1 R)-(-)$-menthyl glyoxylate in this condensation reaction provided the adduct $3 \mathbf{e}$ in $62 \%$ yield as a mixture of diastereomers in 1:1 ratio, as determined by the ${ }^{1} \mathrm{H}-\mathrm{NMR}$ spectrum of the crude product. Condensation of $(S)-(-)-S$-methyl-S-phenylsulfoximine $(S)$-1a with formaldehyde and benzotriazole afforded the enantiopure benzotriazole intermediate $(S)-3 a$ in $69 \%$ yield. Structures of intermediates $3 \mathbf{a}-\mathbf{e}$ are supported by their ${ }^{1} \mathrm{H}$ and ${ }^{13} \mathrm{C}$ NMR spectra and by elemental analysis or high-resolution MS data.

\section{Nucleophilic substitution of 3a-c with organozinc reagents}

Lewis acid $\left(\mathrm{ZnCl}_{2}\right)$ facilitates the loss of the benzotriazolyl anion in $N$-(benzotriazol-1-ylalkyl)sulfoximines 3 to form $N$-methylene-( $\lambda^{6}$-sulfanylidene)iminium ions 4 -I $\leftrightarrow 1$-(methyleneamino)$\lambda^{4}$-sulfonium ions 4-II, which can undergo nucleophilic addition by organozinc reagents. Nucleophilic substitution of the benzotriazolyl group in 3a-c by allyl or aryl groups was achieved by treatment of 3a-c with allyl- or arylzinc reagents prepared in situ by reaction of zinc chloride with the corresponding Grignard reagents. The desired $N$-functionalized sulfoximines 
5a-h were obtained in 41-65\% yields (Scheme 2, Table 2). Reaction of $3 \mathbf{b}$ with benzylzinc chloride gave 5e as a mixture of diastereoisomers in a 2:3 ratio. Attempts to improve the diastereoselectivity by lowering the reaction temperature or increasing the reaction time remained unsuccessful.

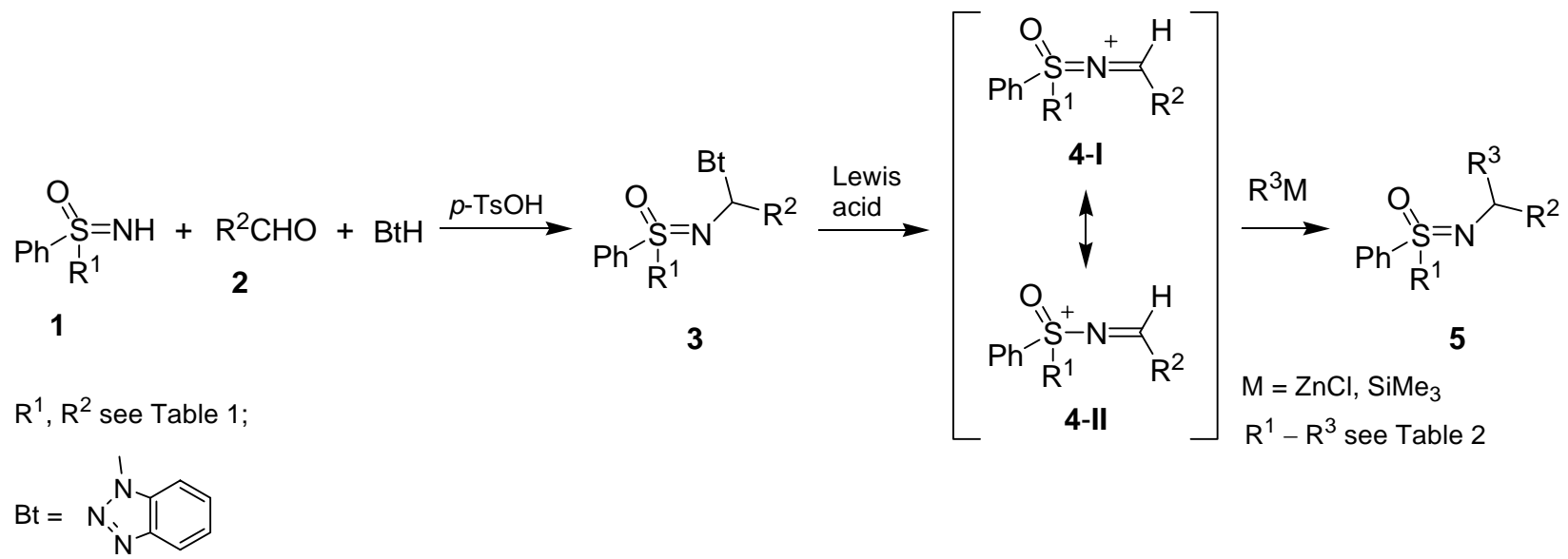

\section{Scheme 2}

Table 1. Preparation of $N$-(benzotriazol-1-ylalkyl)sulfoximines 3a-e

\begin{tabular}{|c|c|c|c|c|c|}
\hline Sulfoximine & Aldehyde & $\mathrm{R}^{1}$ & $\mathrm{R}^{2}$ & Product & Yield [\%] \\
\hline $1 \mathrm{a}$ & $2 a$ & $\mathrm{Me}$ & $\mathrm{H}$ & 3a & 73 \\
\hline $1 a$ & $2 \mathbf{b}$ & $\mathrm{Me}$ & $\mathrm{CO}_{2} \mathrm{Et}$ & $3 \mathbf{b}$ & 82 \\
\hline $1 b$ & $2 \mathbf{a}$ & $\mathrm{Ph}$ & $\mathrm{H}$ & $3 c$ & 78 \\
\hline $1 b$ & $2 \mathbf{b}$ & $\mathrm{Ph}$ & $\mathrm{CO}_{2} \mathrm{Et}$ & 3d & 71 \\
\hline $1 b$ & 2c & $\mathrm{Ph}$ & & $3 \mathbf{e}$ & 62 \\
\hline$(S)-\mathbf{1 a}$ & $2 a$ & $\mathrm{Me}$ & $\mathrm{H}$ & $(S)-\mathbf{3 a}$ & 69 \\
\hline
\end{tabular}

\section{Nucleophilic substitution of 3a-e with allylsilanes}

Lewis acid promoted reactions of allylsilanes with benzotriazole intermediates result in nucleophilic substitution. ${ }^{15}$ Reaction of $3 \mathbf{b}$ with allyltrimethylsilane in the presence of $\mathrm{BF}_{3} \cdot \mathrm{Et}_{2} \mathrm{O}$ gave $N$-substituted sulfoximine $5 \mathbf{i}$ in $64 \%$ yield (Table 2). Similarly, (benzotriazol-1-ylmethyl)diphenylsulfoximine 3c reacted with allyltrimethylsilane or (2-methylpropenyl)trimethylsilane to give $\mathbf{5} \mathbf{j}$ and $\mathbf{5 k}$ in $\mathbf{7 4 \%}$ and $\mathbf{9 2 \%}$ yields, respectively. Bulky intermediates $\mathbf{3 d , e}$, which showed no reactivity towards organozinc reagents, reacted readily with 
allyltrimethylsilane to afford $N$-substituted sulfoximines $5 \mathbf{l}$ and $5 \mathrm{~m}$ in $71 \%$ and $60 \%$ yields, respectively. However, the sulfur chiral center did not exert any diastereoselectivity, and sulfoximine $5 \mathbf{i}$ was obtained as a $1: 1$ mixture of diastereoisomers as determined by ${ }^{1} \mathrm{H}$ and ${ }^{13} \mathrm{C}$ NMR spectra. Use of (R)-(-)-menthyl group as an additional chiral moiety in $\mathbf{3 e}$ also resulted in the formation of a 1:1 mixture of diastereomers $5 \mathrm{~m}$ in $60 \%$ yield upon reaction with allyl(trimethyl)silane. Similarly, reactions of (S)-3a with allyl(trimethyl)silane or 2-methyl-3(trimethyl)silyl-1-propene gave sulfoximines (S)-5a or (S)-5n in 77 and 67\% yields, respectively.

Table 2. Preparation of $N$-substituted sulfoximines $5 \mathbf{a}-\mathbf{n}$

\begin{tabular}{|c|c|c|c|c|c|c|}
\hline $\begin{array}{l}\text { Staring } \\
\text { material }\end{array}$ & Nucleophile & $\mathrm{R}^{1}$ & $\mathrm{R}^{2}$ & $\mathrm{R}^{3}$ & Product & $\begin{array}{c}\text { Yield } \\
{[\%]}\end{array}$ \\
\hline $3 a$ & $\sim \mathrm{ZnCl}$ & $\mathrm{Me}$ & $\mathrm{H}$ & $\sim$ & $5 a$ & 45 \\
\hline $3 a$ & $\mathrm{PhZnCl}$ & $\mathrm{Me}$ & $\mathrm{H}$ & $\mathrm{Ph}$ & $5 \mathbf{b}$ & 45 \\
\hline $3 a$ & $4-\mathrm{ClC}_{6} \mathrm{H}_{4} \mathrm{ZnCl}$ & $\mathrm{Me}$ & $\mathrm{H}$ & $4-\mathrm{ClC}_{6} \mathrm{H}_{4}$ & $5 c$ & 62 \\
\hline $3 a$ & $\mathrm{PhCH}_{2} \mathrm{ZnCl}$ & $\mathrm{Me}$ & $\mathrm{H}$ & $\mathrm{PhCH}_{2}$ & $5 d$ & 65 \\
\hline $3 \mathbf{b}$ & $\mathrm{PhCH}_{2} \mathrm{ZnCl}$ & $\mathrm{Me}$ & $\mathrm{CO}_{2} \mathrm{Et}$ & $\mathrm{PhCH}_{2}$ & $5 e$ & 41 \\
\hline $3 c$ & $4-\mathrm{ClC}_{6} \mathrm{H}_{4} \mathrm{ZnCl}$ & $\mathrm{Ph}$ & $\mathrm{H}$ & $4-\mathrm{ClC}_{6} \mathrm{H}_{4}$ & $5 f$ & 62 \\
\hline $3 c$ & $\mathrm{PhCH}_{2} \mathrm{ZnCl}$ & $\mathrm{Ph}$ & $\mathrm{H}$ & $\mathrm{Ph}$ & $5 g$ & 49 \\
\hline $3 c$ & (2-mesityl) ZnCl & $\mathrm{Ph}$ & $\mathrm{H}$ & 2-mesityl & $5 h$ & 53 \\
\hline $3 \mathbf{b}$ & $\sim \mathrm{SiMe}_{3}$ & $\mathrm{Me}$ & $\mathrm{CO}_{2} \mathrm{Et}$ & & $5 \mathbf{i}$ & 64 \\
\hline $3 c$ & $\mathrm{SiMe}_{3}$ & $\mathrm{Ph}$ & $\mathrm{H}$ & & $5 \mathbf{j}$ & 74 \\
\hline $3 c$ & $\lambda \mathrm{SiMe}_{3}$ & $\mathrm{Ph}$ & $\mathrm{H}$ & & $5 \mathbf{k}$ & 92 \\
\hline 3d & $\sim \mathrm{SiMe}_{3}$ & $\mathrm{Ph}$ & $\mathrm{CO}_{2} \mathrm{Et}$ & & 51 & 71 \\
\hline $3 \mathbf{e}$ & $\mathrm{SiMe}_{3}$ & $\mathrm{Ph}$ & & & $5 \mathrm{~m}$ & 60 \\
\hline$(S)-\mathbf{3 a}$ & $\sim \mathrm{SiMe}_{3}$ & $\mathrm{Me}$ & $\mathrm{H}$ & & $(S)-5 a$ & 77 \\
\hline$(S)-3 \mathbf{a}$ & $\mathrm{diMe}_{3}$ & $\mathrm{Me}$ & $\mathrm{H}$ & $d$ & $(S)-5 n$ & 67 \\
\hline
\end{tabular}

\section{Conclusions}

In summary, we have introduced a general and convenient method for the preparation of $N$ substituted sulfoximines via readily available $N$-(benzotriazol-1-ylmethyl)sulfoximines. 


\section{Experimental Section}

General Procedures. ${ }^{1} \mathrm{H}$ NMR spectra were determined at $300 \mathrm{MHz}$ and ${ }^{13} \mathrm{C} \mathrm{NMR}$ at $75 \mathrm{MHz}$ in $\mathrm{CDCl}_{3}$ (with TMS for ${ }^{1} \mathrm{H}$ and $\mathrm{CDCl}_{3}$ for ${ }^{13} \mathrm{C}$ as the internal reference). HRMS was measured on an AEI-30 mass spectrometer. Tetrahydrofuran (THF) was distilled from Na/benzophenone, dichloromethane was distilled from $\mathrm{CaH}_{2}$ under $\mathrm{N}_{2}$. Sulfoximines 1a, $\mathbf{1 b}$ and $(S)$-1a were prepared according to literature procedures. ${ }^{16}$

\section{General procedure for the preparation of $\boldsymbol{N}$-(benzotriazol-1-ylalkyl)sulfoximines $3 a-e$}

A mixture of aldehyde $2(10 \mathrm{mmol})$, benzotriazole $(1.19 \mathrm{~g}, 10 \mathrm{mmol})$, sulfoximine 1 (10 mmol) and catalytic $p$-TsOH $\left(30 \mathrm{mg}\right.$ ) in toluene was refluxed overnight under $\mathrm{N}_{2}$ in a Dean-Stark apparatus. After removal of water, toluene was evaporated under reduced pressure, and the residue was purified by column chromatography with hexanes/ethyl acetate (from 1:1 to 1:3) to give the desired product 3 .

1-[[[Methyl(oxo)phenyl- $\lambda^{6}$-sulfanylidene]amino]methyl]-1H-1,2,3-benzotriazole (3a). Colorless plates (from hexanes/chloroform); mp 88-89 ${ }^{\circ} \mathrm{C}$; yield $73 \% .{ }^{1} \mathrm{H}$ NMR: $\delta 7.93(\mathrm{~d}, J=8.4 \mathrm{~Hz}$, $1 \mathrm{H}), 7.72-7.68(\mathrm{~m}, 3 \mathrm{H}), 7.53-7.29(\mathrm{~m}, 5 \mathrm{H}), 5.95(\mathrm{~d}, J=13.2 \mathrm{~Hz}, 1 \mathrm{H}), 5.84(\mathrm{~d}, J=13.2 \mathrm{~Hz}, 1 \mathrm{H})$, 3.09 (s, 3H); ${ }^{13} \mathrm{C}$ NMR: $\delta$ 146.0, 139.0, 133.3, 132.5, 129.3, 127.7, 127.1, 123.7, 119.6, 110.3, 57.0, 45.4. Anal. Calcd for $\mathrm{C}_{14} \mathrm{H}_{14} \mathrm{~N}_{4} \mathrm{OS}$ : C, 58.72; H, 4.93; N, 19.57. Found: C, 58.86; H, 4.79; $\mathrm{N}, 19.95$.

Ethyl 2-(1H-1,2,3-benzotriazol-1-yl)-2-[[methyl(oxo)phenyl- $\lambda^{6}$-sulfanylidene]amino]acetate (3b). A 1:1 mixture of diastereomers as determined by ${ }^{1} \mathrm{H}$ NMR spectrum of the crude product. After recrystallization, one isomer was separated and characterized. Colorless plates (from hexanes/chloroform); mp $114-115{ }^{\circ} \mathrm{C}$; yield 82\%. ${ }^{1} \mathrm{H}$ NMR: $\delta$ 8.07-7.99 (m, 1H), 7.74-7.62 (m, $3 \mathrm{H}), 7.51(\mathrm{t}, J=7.4 \mathrm{~Hz}, 1 \mathrm{H}), 7.38(\mathrm{t}, J=8.1 \mathrm{~Hz}, 1 \mathrm{H}), 6.63(\mathrm{~s}, 1 \mathrm{H}), 4.25-4.10(\mathrm{~m}, 2 \mathrm{H}), 3.07$ (s, $3 \mathrm{H}), 1.15$ (t, $J=7.1 \mathrm{~Hz}, 3 \mathrm{H}) .{ }^{13} \mathrm{C}$ NMR: $\delta 167.5,146.5,137.6,133.9,132.1,129.7,128.6,127.2$, 123.9, 119.7, 112.5, 70.3, 62.3, 45.1, 13.8. Anal. Calcd for $\mathrm{C}_{17} \mathrm{H}_{18} \mathrm{~N}_{4} \mathrm{O}_{3} \mathrm{~S}: \mathrm{C}, 56.97 ; \mathrm{H}, 5.06 ; \mathrm{N}$, 15.63. Found: C, 57.31; H, 4.95; N, 15.92 .

1-[I[Oxo(diphenyl)- $\lambda^{6}$-sulfanylidene]amino]methyl]-1H-1,2,3-benzotriazole (3c). White prisms (from hexanes/chloroform); mp 123-124 ${ }^{\circ} \mathrm{C}$; yield 78\%. ${ }^{1} \mathrm{H}$ NMR: $\delta 7.96(\mathrm{~d}, J=8.4 \mathrm{~Hz}, 1 \mathrm{H}$ ), $7.86(\mathrm{~d}, J=7.4 \mathrm{~Hz}, 4 \mathrm{H}), 7.79(\mathrm{~d}, J=8.4 \mathrm{~Hz}, 1 \mathrm{H}), 7.50-7.28(\mathrm{~m}, 8 \mathrm{H}), 6.04(\mathrm{~s}, 2 \mathrm{H}) .{ }^{13} \mathrm{C} \mathrm{NMR}: \delta$ $146.1,140.0,132.9,132.6,129.2,128.0,127.1,123.7,119.6,110.6,57.3$. Anal. Calcd for $\mathrm{C}_{19} \mathrm{H}_{16} \mathrm{~N}_{4} \mathrm{OS}$ : C, 65.50; H, 4.63; N, 16.08. Found: C, 65.80; H, 4.48; N, 16.17.

Ethyl 2-(1H-1,2,3-benzotriazol-1-yl)-2-[[oxo(diphenyl)- $\lambda^{6}$-sulfanylidene]amino]acetate (3d). White prisms (from hexanes/ethyl acetate); mp 86-87 ${ }^{\circ} \mathrm{C}$; yield 71\%. ${ }^{1} \mathrm{H}$ NMR: $\delta$ 8.05-7.96 (m, 4H), 7.67-7.64 (m, 2H), 7.62-7.25 (m, 8H), $6.77(\mathrm{~s}, 1 \mathrm{H}), 4.22(\mathrm{q}, J=7.0 \mathrm{~Hz}, 2 \mathrm{H}), 1.20(\mathrm{t}, J=$ $7.0 \mathrm{~Hz}, 3 \mathrm{H}) .{ }^{13} \mathrm{C}$ NMR: $\delta 167.7,146.4,139.0,133.3,133.0,132.1,129.4,129.1,128.4,128.0$, 127.2, 123.9, 119.6, 112.6, 70.0, 62.4, 13.9. Anal. Calcd for $\mathrm{C}_{22} \mathrm{H}_{20} \mathrm{~N}_{4} \mathrm{O}_{3} \mathrm{~S}: \mathrm{C}, 62.84 ; \mathrm{H}, 4.79 ; \mathrm{N}$, 13.32. Found: C, 62.96; H, 4.77; N, 13.27. 
(1R,2S,5R)-2-Isopropyl-5-methylcyclohexyl 2-(1H-1,2,3-benzotriazol-1-yl)-2-[[diphenyl(oxo)$\lambda^{6}$-sulfanylidene]amino]acetate (3e). Isolated as a 1:1 mixture of diastereomers; colorless oil; yield 62\%. ${ }^{1} \mathrm{H}$ NMR: $\delta$ 8.03-7.94 (m, 4H), 7.75-7.68 (m, 2H), 7.60-7.25 (m, 8H), $6.77(\mathrm{~s}, 0.5 \times 1$ $\mathrm{H}), 6.76(\mathrm{~s}, 0.5 \times 1 \mathrm{H}$, isomer), $4.76(\mathrm{dt}, J=10.9,4.4 \mathrm{~Hz}, 0.5 \times 1 \mathrm{H}), 4.67(\mathrm{dt}, J=10.9,4.4 \mathrm{~Hz}$, $0.5 \times 1 \mathrm{H}), 2.07-1.81(\mathrm{~m}, 2 \mathrm{H}), 1.65-0.73(\mathrm{~m}, 13 \mathrm{H}), 0.58(\mathrm{~d}, J=6.9 \mathrm{~Hz}, 0.5 \times 3 \mathrm{H}), 0.42(\mathrm{~d}, J=6.9$ $\mathrm{Hz}, 0.5 \times 3 \mathrm{H}$, isomer); ${ }^{13} \mathrm{C} \mathrm{NMR}: \delta 167.3$ (167.2), 146.3 (146.3), 139.2 (139.1), 138.9, 133.2, 133.0 (132.9), 132.1 (132.0), 129.3, 129.0 (128.9), 128.5 (128.4), 128.0 (127.9), 127.1 (127.0), 123.8 (123.7), 119.5, 112.7 (112.5), 70.2 (70.1), 46.8 (46.6), 40.2 (40.1), 33.9, 31.2 (31.2), 25.9 (25.4), 23.1 (22.8), 21.8, (21.8), 20.6 (20.4), 16.1 (15.6). HRMS calcd for $\mathrm{C}_{30} \mathrm{H}_{35} \mathrm{~N}_{4} \mathrm{O}_{3} \mathrm{~S}$ : 531.2429, found: 531.2425 .

(S)-1-[[[Methyl(oxo)phenyl- $\lambda^{6}$-sulanylidene]amino]methyl]-1H-1,2,3-benzotriazole (S)-(3a). $[\alpha]_{\mathrm{D}}^{25}=+32.6\left(\mathrm{c} 1.32, \mathrm{CHCl}_{3}\right)$; other data same as 3a.

\section{General procedure for the nucleophilic substitution of 3a-c with organozinc reagents}

A solution of zinc chloride $(1.0 \mathrm{M}, 1.2 \mathrm{~mL}, 1.2 \mathrm{mmol})$ in THF was added to a flask containing a solution of the Grignard reagent $(1.0 \mathrm{M}, 1.2 \mathrm{~mL}, 1.2 \mathrm{mmol})$ in THF $(10 \mathrm{~mL})$ under nitrogen at $0{ }^{\circ} \mathrm{C}$. The reaction mixture was stirred at $25{ }^{\circ} \mathrm{C}$ for $45 \mathrm{~min}$ and then cooled to $0{ }^{\circ} \mathrm{C}$ again, and $\mathrm{N}$-(benzotriazol-1-ylalkyl)sulfoximine $3(1 \mathrm{mmol})$ in THF $(10 \mathrm{~mL})$ was added dropwise. The reaction mixture was stirred overnight at $25{ }^{\circ} \mathrm{C}$, quenched with dil. aqueous $\mathrm{NH}_{4} \mathrm{Cl}$ and extracted with diethyl ether. The organic layer was washed with $\mathrm{NaHCO}_{3}$, brine, dried over $\mathrm{Na}_{2} \mathrm{SO}_{4}$ and then removed. The residue was purified by column chromatography on silica gel using hexanes/ethyl acetate $(1: 2)$ as eluent to afford the desired $N$ - substituted sulfoximines $\mathbf{5 a}-\mathbf{h}$.

(3-Butenylimino)(methyl)oxo(phenyl)- $\lambda^{6}$-sulfane (5a). Colorless oil; yield $45 \%$. ${ }^{1} \mathrm{H}$ NMR: $\delta$ 7.93-7.90 (m, 2H), 7.65-7.54 (m, 3H), 5.90-5.76 (m, 1H), 5.08-4.97 (m, 2H), 3.14-3.00 (m, 4H), 2.90-2.81 (m, 1H), 2.32 (q, $J=7.1 \mathrm{~Hz}, 2 \mathrm{H}) .{ }^{13} \mathrm{C}$ NMR: $\delta 139.5,136.6,132.8,129.4,128.6$, 115.7, 45.1, 43.5, 37.1. Anal. Calcd for $\mathrm{C}_{11} \mathrm{H}_{15} \mathrm{NOS}$ : C, 63.12; H, 7.22; N, 6.69. Found: C, 62.64; H, 7.51; N, 6.89.

(Benzylimino)(methyl)oxo(phenyl)- $\lambda^{6}$-sulfane (5b). ${ }^{17}$ Colorless oil; yield $45 \% .{ }^{1} \mathrm{H}$ NMR: $\delta$ 7.95-7.92 (m, 2H), 7.64-7.53 (m, 3H), 7.37-7.16 (m, 5H), 4.21 (d, $J=14.3 \mathrm{~Hz}, 1 \mathrm{H}), 3.97$ (d, $J=$ $14.3 \mathrm{~Hz}, 1 \mathrm{H}), 3.14$ (s, 3H). ${ }^{13} \mathrm{C}$ NMR: $\delta$ 141.2, 139.4, 132.9, 129.4, 128.6, 128.2, 127.6, 126.5, 47.3, 45.3. Anal. Calcd for $\mathrm{C}_{14} \mathrm{H}_{15} \mathrm{NOS}$ : C, 68.54; H, 6.16; N, 5.71. Found: $\mathrm{C}, 68.07$; H, 6.32; N, 5.92 .

[(4-Chlorobenzyl)imino](methyl)oxo(phenyl)- $\lambda^{6}$-sulfane $\mathbf{( 5 c )} .^{9 \mathrm{~b}}$ White prisms (from hexanes /ethyl acetate); mp 61-62 ${ }^{\circ} \mathrm{C}$; yield $62 \% .{ }^{1} \mathrm{H}$ NMR: $\delta 7.93-7.90(\mathrm{~m}, 2 \mathrm{H}), 7.66-7.52(\mathrm{~m}, 3 \mathrm{H})$, $7.31-7.23(\mathrm{~m}, 4 \mathrm{H}), 4.15$ (d, $J=14.6 \mathrm{~Hz}, 1 \mathrm{H}), 3.93(\mathrm{~d}, J=14.6 \mathrm{~Hz}, 1 \mathrm{H}), 3.15(\mathrm{~s}, 3 \mathrm{H}) .{ }^{13} \mathrm{C}$ NMR: $\delta$ 139.7, 139.3, 133.0, 132.2, 129.5, 128.9, 128.6, 128.3, 46.7, 45.3. Anal. Calcd for $\mathrm{C}_{14} \mathrm{H}_{14}$ CINOS: C, 60.10; H, 5.04; N, 5.01. Found: C, 60.06; H, 5.12; N, 4.97.

Methyl(oxo)(phenethylimino)(phenyl)- $\lambda^{6}$-sulfane (5d). Colorless oil; yield $65 \%$. ${ }^{1} \mathrm{H}$ NMR: $\delta$ 7.79-7.76 (m, 2H), 7.61-7.48 (m, 3H), 7.27-7.16 (m, 5H), 3.28-3.19 (m, 1H), 3.06-2.96 (m, 
4H), 2.90-2.85 (m, 2H). ${ }^{13} \mathrm{C}$ NMR: $\delta$ 140.4, 139.4, 132.8, 129.3, 128.9, 128.6, 128.2, 125.9, 45.8, 45.1, 39.4. HRMS calcd for $\mathrm{C}_{15} \mathrm{H}_{18} \mathrm{NOS}$ : 260.1109, found: 260.1107 .

Ethyl 2-[[methyl(oxo)phenyl- $\lambda^{6}$-sulfanylidene]amino]-3-phenylpropanoate (5e). Isolated as a mixture of diastereomers in 2:3 ratio; colorless oil; yield $41 \% .{ }^{1} \mathrm{H}$ NMR: $\delta 7.91-7.89(\mathrm{~m}, 1 \mathrm{H})$, 7.60-7.50 (m, 2H), 7.32-7.17 (m, 7H), 4.20-3.60 (m, 3H), 3.20-2.90 (m, 5H), 1.23 (t, J= 7.2 Hz, $0.4 \times 3 \mathrm{H}$, diastereomer 1$), 1.02(\mathrm{t}, J=7.1 \mathrm{~Hz}, 0.6 \times 3 \mathrm{H}$, diastereomer 2$) .{ }^{13} \mathrm{C} \mathrm{NMR}: \delta 173.6$ (172.8), 138.3 (137.8), 133.0 (132.7), 129.9, 129.6, 129.2, 129.1, 128.5, 128.4, 128.1, 126.1, 60.8 (60.4), 59.4 (58.3), 45.2 (45.1), 42.3 (41.4), 14.0 (13.9). Anal. Calcd for $\mathrm{C}_{18} \mathrm{H}_{21} \mathrm{NO}_{3} \mathrm{~S}: \mathrm{C}, 65.23$; H, 6.39; N, 4.23. Found: C, 65.41; H, 6.52; N, 4.49.

[(4-Chlorobenzyl)imino](oxo)diphenyl- $\lambda^{6}$-sulfane (5f). Colorless oil; yield $62 \%$. ${ }^{1} \mathrm{H}$ NMR: $\delta$ 8.01-7.98 (m, 4H), 7.53-7.45 (m, 6H), $7.38(\mathrm{~d}, J=8.4 \mathrm{~Hz}, 2 \mathrm{H}), 7.28(\mathrm{~d}, J=8.5 \mathrm{~Hz}, 2 \mathrm{H}), 4.24$ (s, 2H). ${ }^{13} \mathrm{C}$ NMR: $\delta 140.5,140.1,132.5,132.1,129.2,128.8,128.5,128.3,46.5$. Anal. Calcd for $\mathrm{C}_{19} \mathrm{H}_{16} \mathrm{ClNOS}$ : C, 66.76; H, 4.72. Found: C, 66.39; H, 4.96.

(Benzylimino)(oxo)diphenyl- $\lambda^{6}$-sulfane (5g). ${ }^{18}$ Colorless oil; yield 49\%. ${ }^{1} \mathrm{H}$ NMR: $\delta$ 8.03-8.00 (m, 4H), 7.53-7.44 (m, 8H), $7.32(\mathrm{t}, J=7.1 \mathrm{~Hz}, 2 \mathrm{H}), 7.25-7.19(\mathrm{~m}, 1 \mathrm{H}), 4.28(\mathrm{~s}, 2 \mathrm{H}) .{ }^{13} \mathrm{C}$ NMR: $\delta 141.5,140.7,132.4,129.1,128.6,128.2,127.4,126.4$, 47.1. Anal. Calcd for $\mathrm{C}_{19} \mathrm{H}_{17} \mathrm{NOS}$ : C, 74.23; H, 5.57; N, 4.56. Found: C, 74.13; H, 5.59; N, 4.62 .

[(Mesitylmethyl)imino](oxo)diphenyl- $\lambda^{\mathbf{6}}$-sulfane (5h). White microcrystals (from ethyl acetate/hexanes); mp $97{ }^{\circ} \mathrm{C}$; yield 53\%. ${ }^{1} \mathrm{H}$ NMR: $\delta$ 7.96-7.93 (m, 4H), 7.48-7.42 (m, 6H), 6.78 $(\mathrm{s}, 2 \mathrm{H}), 4.20(\mathrm{~s}, 2 \mathrm{H}), 2.35(\mathrm{~s}, 6 \mathrm{H}), 2.23(\mathrm{~s}, 3 \mathrm{H}) .{ }^{13} \mathrm{C}$ NMR: $\delta 141.3,136.9,136.1,134.5,132.1$, 128.9, 128.8, 128.3, 40.7, 20.8, 19.6. Anal. Calcd for $\mathrm{C}_{22} \mathrm{H}_{23} \mathrm{NOS}$ : C, 75.61; H, 6.63; N, 4.01. Found: C, 75.29; H, 6.75; N, 4.01.

\section{General procedure for the nucleophilic substitution of 3a-e with allyl(trimethyl)silanes}

To a mixture of $N$-(benzotriazol-1-ylalkyl)sulfoximine 3 (1 mmol) and allyl(trimethyl)silane (1 $\mathrm{mmol})$ in $\mathrm{CH}_{2} \mathrm{Cl}_{2}(20 \mathrm{~mL})$ was added $\mathrm{BF}_{3} \cdot \mathrm{Et}_{2} \mathrm{O}(2 \mathrm{mmol})$ at $0{ }^{\circ} \mathrm{C}$. The reaction mixture was stirred at $0{ }^{\circ} \mathrm{C}$ for $2 \mathrm{~h}$ and then at room temperature for $18 \mathrm{~h}$. The reaction was quenched by aqueous $\mathrm{NaHCO}_{3}$ solution and extracted with $\mathrm{CH}_{2} \mathrm{Cl}_{2}$. The combined solvent extract was dried over anhydrous $\mathrm{Na}_{2} \mathrm{SO}_{4}$ and removed in vacuo. The residue was purified by column chromatography with hexanes/ethyl acetate $(2: 1)$ as eluent to give the desired $N$-substituted sulfoximine 5.

Ethyl 2-[[methyl(oxo)phenyl- $\lambda^{\mathbf{6}}$-sulfanylidene]amino]-4-pentenoate (5i). Isolated as a $1: 1$ mixture of diastereomers; colorless oil; yield 64\%. ${ }^{1} \mathrm{H}$ NMR: $\delta$ 7.96-7.92 (m, 2H), 7.67-7.52 (m, $3 \mathrm{H}), 5.93-5.70(\mathrm{~m}, 1 \mathrm{H}), 5.13-5.02(\mathrm{~m}, 2 \mathrm{H}), 4.18(\mathrm{q}, J=7.1 \mathrm{~Hz}, 0.5 \times 2 \mathrm{H}$, diastereomer 1), $4.12-$ $3.91(\mathrm{~m}, 0.5 \times 2 \mathrm{H}$, diastereomer 2$), 3.76(\mathrm{t}, J=6.5 \mathrm{~Hz}, 0.5 \times 1 \mathrm{H}$, diastereomer 1$), 3.69(\mathrm{t}, J=6.7$ $\mathrm{Hz}, 0.5 \times 1 \mathrm{H}$, diastereomer 2), $3.15(\mathrm{~s}, 3 \mathrm{H}), 2.64-2.40(\mathrm{~m}, 2 \mathrm{H}), 1.27(\mathrm{t}, J=7.1 \mathrm{~Hz}, 0.5 \times 3 \mathrm{H}$, diastereomer 1), $1.15\left(\mathrm{t}, J=7.1 \mathrm{~Hz}, 0.5 \times 3 \mathrm{H}\right.$, diastereomer 2 ). ${ }^{13} \mathrm{C}$ NMR: $\delta 173.2,172.7$ (other diastereomer), 139.7, 139.4 (other diastereomer), 134.1, 134.0 (other diastereomer), 132.9, 129.2, 128.5, 128.3 (other diastereomer), 117.4, 117.3 (other diastereomer), 60.5, 60.3 (other diastereomer), 56.9, 56.4 (other diastereomer), 45.4, 45.0 (other diastereomer), 40.4, 39.5 (other 
diastereomer), 14.04, 13.98 (other diastereomer). Anal. Calcd for $\mathrm{C}_{14} \mathrm{H}_{19} \mathrm{NO}_{3} \mathrm{~S}$ : C, 59.76; H, $6.81 ; \mathrm{N}, 4.98$. Found: C, 60.17; H, 7.18; N, 4.92.

(3-Butenylimino)(oxo)diphenyl- $\lambda^{6}$-sulfane (5j). Colorless oil; yield $74 \%$. ${ }^{1} \mathrm{H}$ NMR: $\delta$ 8.00$7.96(\mathrm{~m}, 4 \mathrm{H}), 7.53-7.43(\mathrm{~m}, 6 \mathrm{H}), 5.97-5.84(\mathrm{~m}, 1 \mathrm{H}), 5.13-4.99(\mathrm{~m}, 2 \mathrm{H}), 3.12$ (t, $J=7.3 \mathrm{~Hz}$, 2H), 2.46-2.39 (m, 2H). ${ }^{13} \mathrm{C}$ NMR: $\delta 140.7,136.8,132.2,129.0,128.5,115.7,43.5,37.3$. Anal. Calcd for $\mathrm{C}_{16} \mathrm{H}_{17} \mathrm{NOS}$ : C, 70.81; H, 6.31; N, 5.16. Found: C, 71.16; H, 6.47; N, 5.36.

[(3-Methyl-3-butenyl)imino](oxo)diphenyl- $\lambda^{6}$-sulfane (5k). Colorless oil; yield $92 \%$. ${ }^{1} \mathrm{H}$ NMR: $\delta$ 7.99-7.96 (m, 4H), 7.50-7.43 (m, 6H), 4.75-4.74 (m, 2H), 3.17 (t, J=7.6 Hz, 2H), 2.39 $(\mathrm{t}, J=7.6 \mathrm{~Hz}, 2 \mathrm{H}), 1.71(\mathrm{~s}, 3 \mathrm{H}) .{ }^{13} \mathrm{C} \mathrm{NMR}: \delta 144.2,140.8,132.2,129.0,128.5,110.9,42.5$, 41.2, 22.7. Anal. Calcd for $\mathrm{C}_{17} \mathrm{H}_{19} \mathrm{NOS}$ : C, 71.54; H, 6.71; N, 4.91. Found: C, 71.37; H, 6.78; N, 4.80 .

Ethyl 2-[[oxo(diphenyl)- $\lambda^{6}$-sulfanylidene]amino]-4-pentenoate (5l). Colorless oil; yield $71 \%$. ${ }^{1} \mathrm{H}$ NMR: $\delta$ 8.04-7.96 (m, 4H), 7.54-7.42 (m, 6H), 5.98-5.84 (m, 1H), 5.17-5.06 (m, 2H), 4.19$4.06(\mathrm{~m}, 2 \mathrm{H}), 3.83(\mathrm{t}, J=6.7 \mathrm{~Hz}, 1 \mathrm{H}), 2.67-2.60(\mathrm{~m}, 2 \mathrm{H}), 1.23(\mathrm{t}, J=7.0 \mathrm{~Hz}, 3 \mathrm{H}) .{ }^{13} \mathrm{C} \mathrm{NMR}: \delta$ 173.0, 140.3, 134.3, 132.4, 132.4, 128.9, 128.9, 128.6, 128.3, 117.3, 60.5, 56.9, 40.1, 14.1. Anal. Calcd for $\mathrm{C}_{19} \mathrm{H}_{21} \mathrm{NO}_{3} \mathrm{~S}: \mathrm{C}, 66.45 ; \mathrm{H}, 6.16 ; \mathrm{N}, 4.08$. Found: C, 66.56; H, 6.28; N, 4.28.

(1R,2S,5R)-2-Isopropyl-5-methylcyclohexyl $\quad 2$-[[oxo(diphenyl)- $\lambda^{6}$-sulfanylidene]amino]-4pentenoate (5m). Isolated as a 1:1 mixture of diasteromers; colorless oil; yield $60 \%$. ${ }^{1} \mathrm{H}$ NMR: $\delta$ 8.08-8.04 (m, 2H), 7.96-7.92 (m, 2H), 7.54-7.42 (m, 6H), 6.01-5.83 (m, 1H), 5.15-5.06 (m, 2H), 4.78-4.67 (m, 1H), $3.80(\mathrm{t}, J=6.7 \mathrm{~Hz}, 1 \mathrm{H}), 2.71-2.54(\mathrm{~m}, 2 \mathrm{H}), 2.06-1.78(\mathrm{~m}, 2 \mathrm{H}), 1.69-$ $1.64(\mathrm{~m}, 2 \mathrm{H}), 1.55-1.35(\mathrm{~m}, 2 \mathrm{H}), 1.10-0.84(\mathrm{~m}, 9 \mathrm{H}), 0.78-0.72(\mathrm{~m}, 3 \mathrm{H}) .{ }^{13} \mathrm{C} \mathrm{NMR}: \delta 172.7$ (172.6), 140.6, 140.4 (140.3), 134.5 (134.4), 132.5 (132.4), 129.1 (129.0), 129.0, 128.9 (128.8), 128.5 (128.4), 117.4 (117.3), 74.4, 57.5 (57.3), 46.9 (46.8), 40.7 (40.5), 40.4 (40.3), 34.1, 31.3, 25.8 (25.7), 23.2 (22.9), 22.0, 20.8 (20.7), 16.1 (15.8). Anal. Calcd for $\mathrm{C}_{27} \mathrm{H}_{35} \mathrm{NO}_{3} \mathrm{~S}: \mathrm{C}, 71.49 ; \mathrm{H}$, 7.78; N, 3.09. Found: C, 71.33; H, 7.99; N, 3.24.

(S)-(3-Butenylimino)(methyl)oxo(phenyl)- $\lambda^{6}$-sulfane $[(\boldsymbol{S})-5 \mathrm{a}] \cdot[\alpha]_{\mathrm{D}}^{25}=+136.9$ (c 2.08, $\mathrm{CHCl}_{3}$ ). Other data same as $\mathbf{6 a}$.

(S)-Methyl[(3-methyl-3-butenyl)imino]oxo(phenyl)- $\lambda^{\mathbf{6}}$-sulfane [(S)-5n]. Colorless oil; yield 67\%. $[\alpha]_{\mathrm{D}}^{25}=+130.6$ (c 1.75, $\left.\mathrm{CHCl}_{3}\right) ;{ }^{1} \mathrm{H}$ NMR: $\delta$ 7.94-7.90 (m, 2H), 7.62-7.54 (m, 3H), 4.72 $(\mathrm{s}, 1 \mathrm{H}), 4.68(\mathrm{~s}, 1 \mathrm{H}), 3.15-3.05(\mathrm{~m}, 4 \mathrm{H}), 2.95-2.86(\mathrm{~m}, 1 \mathrm{H}), 2.29(\mathrm{t}, J=7.7 \mathrm{~Hz}, 2 \mathrm{H}), 1.69$ (s, 3H). ${ }^{13} \mathrm{C}$ NMR: $\delta$ 144.0, 139.6, 132.8, 129.4, 128.6, 110.8, 45.2, 42.5, 40.9, 22.7. Anal. Calcd for $\mathrm{C}_{12} \mathrm{H}_{17} \mathrm{NOS}$ : C, 64.53; H, 7.67; N, 6.27. Found: C, 64.16; H, 7.97; N, 6.23.

\section{References}

1. Alker, D.; Kemp, J. E. G.; Cross, P. E. PCT Int. Appl. 9110648, 1991; Chem. Abstr. 1991, 115, 207864.

2. Hellberg, M. R.; Shanklin, J. R., Jr. U.S. Pat. 5140026, 1992; Chem. Abstr. 1993, 118, 147299. 
3. Griffith, O. W.; Anderson, M. E.; Meister, A. J. Biol. Chem. 1979, 254, 1205.

4. Harvison, P. J.; Kalman, T. I. J. Med. Chem. 1992, 35, 1227.

5. (a) Johnson, C. R. Acc. Chem. Res. 1973, 6, 341. (b) Reggelin, M.; Zur, C. Synthesis 2000, 1. (c) Craig, D.; Geach, N. J.; Sardharwala, F. E.; White, A. J. P.; Williams, D. J. Arkivoc 2002, (vi), 104.

6. Johnson, C. R.; Schroeck, C. W.; Shanklin, J. R. J. Am. Chem. Soc. 1973, 95, 7424.

7. (a) Bolm, C.; Hildebrand, J. P. Tetrahedron Lett. 1998, 39, 5731. (b) Bolm, C.; Simic, O. J. Am. Chem. Soc. 2001, 123, 3830. (c) Bolm, C.; Martin, M.; Gibson, L. Synlett 2002, 832.

8. Johnson, C. R.; Rigau, J. J.; Haake, M.; McCants Jr. D.; Keiser, J. E.; Gertsema, A. Tetrahedron Lett. 1968, 3719.

9. (a) Johnson, C. R.; Lavergne, O. M. J. Org. Chem. 1993, 58, 1922. (b) Bolm, C.; Hackenberger, C. P. R.; Simic, O.; Verrucci, M.; Muller, D.; Bienewald, F. Synthesis 2002, 879.

10. (a) Katritzky, A. R.; Yannakopoulou, K.; Lue, P.; Rasala, D.; Urogdi, L. J. Chem. Soc., Perkin Trans. 1 1989, 225. (b) Katritzky, A. R.; Fan, W.-Q. J. Fluorine Chem. 1991, 51, 33.

11. (a) Katritzky, A. R.; Drewniak, M.; Lue, P. J. Org. Chem. 1988, 53, 5854. (b) Katritzky, A. R.; Urogdi, L. J. Chem. Soc., Perkin Trans. 1 1990, 1853.

12. Katritzky, A. R.; Denisko, O.; Lang, H. Tetrahedron 1995, 51, 8703.

13. Katritzky, A. R.; Oniciu, D. C.; Ghiviriga, I. Synth. Commun. 1997, 27, 907.

14. (a) Katritzky, A. R. J. Heterocycl. Chem. 1999, 36, 1501. (b) Katritzky, A. R.; Lan, X.; Yang, J. Z.; Denisko, O. V. Chem. Rev. 1998, 98, 409.

15. Katritzky, A. R.; Qiu, G.; He, H.-Y.; Yang, B. J. Org. Chem. 2000, 65, 3683.

16. Misani, F.; Fair, T. W.; Reiner, L. J. Am. Chem. Soc. 1951, 73, 459.

17. Johnson, C. R.; Lavergne, O. M. J. Org. Chem. 1993, 58, 1922.

18. Hoffmann, H.; Gescher, A. J. Electroanal. Chem. 1976, 74, 329. 